\title{
COMPONENT IMPORTANCE IN COHERENT SYSTEMS WITH EXCHANGEABLE COMPONENTS
}

\author{
SERKAN ERYILMAZ, ${ }^{*}$ Atilim University
}

\begin{abstract}
This paper is concerned with the Birnbaum importance measure of a component in a binary coherent system. A representation for the Birnbaum importance of a component is obtained when the system consists of exchangeable dependent components. The results are closely related to the concept of the signature of a coherent system. Some examples are presented to illustrate the results.
\end{abstract}

Keywords: Component importance; exchangeability; order statistics; signature

2010 Mathematics Subject Classification: Primary 62N05

Secondary $60 \mathrm{~K} 10$

\section{Introduction}

Measuring the importance of a component in a system is an important problem in reliability engineering. Various reliability importance measures have been defined and studied in the literature; see Birnbaum (1969), Barlow and Proschan (1975), Butler (1977), Natvig (1979), (1985), Bergman (1985), Xie (1987), Xie and Bergman (1991), Iyer (1992), Boland and ElNeweihi (1995), Hwang (2001), Andrews (2008), Natvig and Gåsemyr (2009), and Natvig (2011). For a lucid review of the topic, see Kuo and Zhu (2012) and Zhu and Kuo (2014).

Consider a binary coherent system consisting of $n$ binary components. Let $X_{i}(t)$ denote the state of the $i$ th component at time $t, i=1, \ldots, n$, and define

$$
X_{i}(t)= \begin{cases}1 & \text { if the } i \text { th component functions at time } t \\ 0 & \text { if the } i \text { th component has failed at time } t .\end{cases}
$$

If $T_{i}$ denotes the lifetime of the $i$ th component, then $\left\{X_{i}(t)=1\right\} \equiv\left\{T_{i}>t\right\}, i=1, \ldots, n$. Let $\phi$ denote the structure function of the system. Then the state of the system at time $t$ is defined by

$$
\phi\left(X_{1}(t), \ldots, X_{n}(t)\right)= \begin{cases}1 & \text { if the system is functioning at time } t \\ 0 & \text { if the system has failed at time } t\end{cases}
$$

Let $T$ denote the lifetime of the system. Birnbaum (1969) defined the importance of the $i$ th component at time $t$ by

$$
I_{i}(t)=\mathbb{P}\left\{T>t \mid T_{i}>t\right\}-\mathbb{P}\left\{T>t \mid T_{i} \leq t\right\} \quad \text { for } i=1, \ldots, n .
$$

The importance measure defined by (1) is included in the class of lifetime importance measures. Such an importance measure is considered when a system and components have long-term or infinite service missions, and depends on both the positions of the components in the system and component lifetime distributions; see Kuo and Zhu (2012).

Received 3 June 2014; revision received 11 August 2014.

* Postal address: Department of Industrial Engineering, Atilim University, Ankara, Turkey.

Email address: serkan.eryilmaz@atilim.edu.tr 
In this paper we study the Birnbaum importance measure for coherent systems consisting of exchangeable components. A sequence on lifetimes $T_{1}, \ldots, T_{n}$ is exchangeable if

$$
\mathbb{P}\left\{T_{1} \leq t_{1}, \ldots, T_{n} \leq t_{n}\right\}=\mathbb{P}\left\{T_{\pi(1)} \leq t_{1}, \ldots, T_{\pi(n)} \leq t_{n}\right\}
$$

for any permutation $\pi=(\pi(1), \ldots, \pi(n))$ of $\{1, \ldots, n\}$, i.e. the joint distribution (or survival function) of $T_{1}, \ldots, T_{n}$ is symmetric in $t_{1}, \ldots, t_{n}$. Exchangeability means that the components have identical distributions but it allows for a certain type of dependence. For example, if the components are positively exchangeable dependent then the failure of one component causes failure of another component to become more likely. For recent discussions on systems with exchangeable components, see Navarro et al. (2008), Navarro and Spizzichino (2010), Navarro and Rubio (2011), and Eryilmaz et al. (2011).

Our method for studying $I_{i}(t)$ is closely related to the concept of the system signature. For a coherent system with lifetime $T=\phi\left(T_{1}, \ldots, T_{n}\right)$ the signature is defined by the vector $\boldsymbol{s}=\left(s_{1}(n), \ldots, s_{n}(n)\right)$ with

$$
=\frac{\text { number of orderings for which the } m \text { th failure causes system failure }}{n !}, \quad i=1, \ldots, n
$$

and $\sum_{m=1}^{n} s_{m}(n)=1$; see, e.g. Samaniego (2007). In words, $s_{m}(n)$ is the proportion of permutations among the $n$ ! equally likely permutations of component lifetimes that result in a minimal cut set failure when $m$ components break down. Therefore, we have $s_{m}(n)=\mathbb{P}\{T=$ $\left.T_{m: n}\right\}, m=1, \ldots, n$, where $T_{m: n}$ is the $m$ th order statistic among $T_{1}, \ldots, T_{n}$. For a sequence of independent and identical lifetimes $T_{1}, \ldots, T_{n}$, Samaniego (1985) showed that the survival function of $T$ can be represented as

$$
\mathbb{P}\{T>t\}=\sum_{m=1}^{n} s_{m}(n) \mathbb{P}\left\{T_{m}: n>t\right\} .
$$

This representation has been extended to the case of exchangeable components by Navarro et al. (2005), (2008), and Navarro and Rychlik (2007). For some recent works on signature-based reliability analysis, see Navarro et al. (2013), Parvardeh and Balakrishnan (2013), Eryilmaz (2013), Zarazadeh et al. (2014), and Triantafyllou and Koutras (2014).

The signature of a coherent system can be computed from

$$
s_{m}(n)=a_{n-m+1}(n)-a_{n-m}(n), \quad m=1,2, \ldots, n,
$$

where

$$
a_{m}(n)=\frac{r_{m}(n)}{\left(\begin{array}{c}
n \\
m
\end{array}\right)}, \quad i=1,2, \ldots, n,
$$

and $r_{m}(n)$ is the number of path sets of size $m$; see Boland (2001).

In Section 2 we obtain a representation for $I_{i}(t)$ when $T_{1}, \ldots, T_{n}$ are exchangeable dependent. The measure $I_{i}(t)$ is represented in terms of the conditional probabilities $\mathbb{P}\left\{T_{m}: n>\right.$ $\left.t \mid T_{i}>t\right\}$ and $\mathbb{P}\left\{T_{m: n}>t \mid T_{i} \leq t\right\}$ with well-defined coefficients which depend on the structure of a coherent system.

In Theorem 1 we obtain the following equation:

$$
I_{i}(t)=\sum_{m=1}^{n}\left[s_{m}^{+i}(n) \mathbb{P}\left\{T_{m: n}>t \mid T_{i}>t\right\}-s_{m}^{-i}(n) \mathbb{P}\left\{T_{m: n}>t \mid T_{i} \leq t\right\}\right],
$$


where the vectors $\boldsymbol{s}^{+i}=\left(s_{1}^{+i}(n), \ldots, s_{n}^{+i}(n)\right)$ and $\boldsymbol{s}^{-i}=\left(s_{1}^{-i}(n), \ldots, s_{n}^{-i}(n)\right)$ of coefficients depend only on the structure of the system. Some properties of these coefficients are revealed and they are computed for all coherent systems with $n=3$ and $n=4$ exchangeable components. In Section 3 we consider the series system whose lifetime is expressed as $T_{*}=\min \left(\phi_{1}\left(T_{1}, \ldots, T_{n_{1}}\right), \phi_{2}\left(T_{n_{1}+1}, \ldots, T_{n_{1}+n_{2}}\right)\right)$. We provide a method for computing the vectors $\boldsymbol{s}^{+i}$ and $\boldsymbol{s}^{-i}$ for the system with lifetime $T_{*}$ based on the signature vectors $\boldsymbol{p}$ and $\boldsymbol{q}$, and $\boldsymbol{p}^{+i}$ and $\boldsymbol{q}^{+i}$ of the disjoint structures $\phi_{1}$ and $\phi_{2}$.

\section{A representation for the Birnbaum measure}

Define $r_{m}^{+i}(n)$ to be the number of path sets of size $m$ including component $i$ in a coherent system of order $n$. Similarly, let $r_{m}^{-i}(n)$ denote the number of path sets of size $m$ which do not contain the component $i$ in a coherent system of order $n$. If $r_{m}(n)$ is the number of path sets of size $m$ then clearly, we have

$$
r_{m}(n)=r_{m}^{+i}(n)+r_{m}^{-i}(n)
$$

with $r_{0}^{+i}(n)=0, r_{n}^{+i}(n)=1, r_{0}^{-i}(n)=0$, and $r_{n}^{-i}(n)=0$.

For an illustration, consider the system with structure function

$$
\phi\left(x_{1}, x_{2}, x_{3}\right)=\max \left(x_{2}, \min \left(x_{1}, x_{3}\right)\right) .
$$

The corresponding path sets are $\{2\},\{1,2\},\{1,3\},\{2,3\}$, and $\{1,2,3\}$. For $i=1$, we have $r_{1}^{+1}(n)=0, r_{1}^{-1}(n)=1, r_{2}^{+1}(n)=2, r_{2}^{-1}(n)=1, r_{3}^{+1}(n)=1, r_{3}^{-1}(n)=0$.

In the following theorem, we obtain a representation for the Birnbaum importance of the $i$ th component in an arbitrary coherent system of exchangeable components.

Theorem 1. For a coherent system consisting of $n$ exchangeable components with lifetimes $T_{1}, \ldots, T_{n}$,

$$
I_{i}(t)=\sum_{m=1}^{n}\left[s_{m}^{+i}(n) \mathbb{P}\left\{T_{m: n}>t \mid T_{i}>t\right\}-s_{m}^{-i}(n) \mathbb{P}\left\{T_{m: n}>t \mid T_{i} \leq t\right\}\right],
$$

where

$$
\begin{aligned}
& s_{m}^{+i}(n)=\frac{r_{n-m+1}^{+i}(n)}{\left(\begin{array}{c}
n-1 \\
n-m
\end{array}\right)}-\frac{r_{n-m}^{+i}(n)}{\left(\begin{array}{c}
n-1 \\
n-m-1
\end{array}\right)} \text { for } m=1, \ldots, n, \\
& s_{m}^{-i}(n)=\frac{r_{n-m+1}^{-i}(n)}{\left(\begin{array}{c}
n-1 \\
n-m+1
\end{array}\right)}-\frac{r_{n-m}^{-i}(n)}{\left(\begin{array}{c}
n-1 \\
n-m
\end{array}\right)} \text { for } m=2, \ldots, n
\end{aligned}
$$

with $s_{1}^{-i}(n)=0$.

Proof. Consider first the conditional probability $\mathbb{P}\left\{T>t \mid T_{i}>t\right\}$. Let $S_{n}(t)$ denote the total number of working components in a system at time $t$. Then

$$
\mathbb{P}\left\{T>t \mid T_{i}>t\right\}=\sum_{m=1}^{n} \mathbb{P}\left\{T>t \mid T_{i}>t, S_{n}(t)=m\right\} \mathbb{P}\left\{S_{n}(t)=m \mid T_{i}>t\right\} .
$$

Clearly, we have

$$
\begin{gathered}
\mathbb{P}\left\{T>t \mid T_{i}>t, S_{n}(t)=m\right\}=\frac{r_{m}^{+i}(n)}{\left(\begin{array}{c}
n-1 \\
m-1
\end{array}\right)}, \\
\mathbb{P}\left\{S_{n}(t)=m \mid T_{i}>t\right\}=\mathbb{P}\left\{T_{n-m+1: n}>t \mid T_{i}>t\right\}-\mathbb{P}\left\{T_{n-m: n}>t \mid T_{i}>t\right\} .
\end{gathered}
$$


Therefore,

$$
\begin{aligned}
P\left\{T>t \mid T_{i}>t\right\} & =\sum_{m=1}^{n} \frac{r_{m}^{+i}(n)}{\left(\begin{array}{c}
n-1 \\
m-1
\end{array}\right)}\left[\mathbb{P}\left\{T_{n-m+1: n}>t \mid T_{i}>t\right\}-\mathbb{P}\left\{T_{n-m: n}>t \mid T_{i}>t\right\}\right] \\
& =\sum_{m=1}^{n}\left[\frac{r_{n-m+1}^{+i}(n)}{\left(\begin{array}{c}
n-1 \\
n-m
\end{array}\right)}-\frac{r_{n-m}^{+i}(n)}{\left(\begin{array}{c}
n-1 \\
n-m-1
\end{array}\right)}\right] \mathbb{P}\left\{T_{m: n}>t \mid T_{i}>t\right\}
\end{aligned}
$$

On the other hand,

$$
\mathbb{P}\left\{T>t \mid T_{i} \leq t\right\}=\sum_{m=1}^{n-1} \mathbb{P}\left\{T>t \mid T_{i} \leq t, S_{n}(t)=m\right\} \mathbb{P}\left\{S_{n}(t)=m \mid T_{i} \leq t\right\} .
$$

Because

$$
\mathbb{P}\left\{T>t \mid T_{i} \leq t, S_{n}(t)=m\right\}=\frac{r_{m}^{-i}(n)}{\left(\begin{array}{c}
n-1 \\
m
\end{array}\right)},
$$

we have

$$
\mathbb{P}\left\{T>t \mid T_{i} \leq t\right\}=\sum_{m=2}^{n}\left[\frac{r_{n-m+1}^{-i}(n)}{\left(\begin{array}{c}
n-1 \\
n-m+1
\end{array}\right)}-\frac{r_{n-m}^{-i}(n)}{\left(\begin{array}{c}
n-1 \\
n-m
\end{array}\right)}\right] \mathbb{P}\left\{T_{m: n}>t \mid T_{i} \leq t\right\} .
$$

Thus, the proof is completed by substituting (5) and (6) into (1).

For a sequence of exchangeable lifetimes $T_{1}, \ldots, T_{n}$, the expressions for the conditional probabilities $\mathbb{P}\left\{T_{m: n}>t \mid T_{i}>t\right\}$ and $\mathbb{P}\left\{T_{m: n}>t \mid T_{i} \leq t\right\}$ are presented in the following lemma.

Lemma 1. For exchangeable lifetimes $T_{1}, \ldots, T_{n}$,

$$
\begin{aligned}
& \mathbb{P}\left\{T_{m: n}>t \mid T_{i}>t\right\} \\
& \quad=\frac{1}{\mathbb{P}\left\{T_{i}>t\right\}} \sum_{j=n-m}^{n-1} \sum_{s=0}^{n-j-1}(-1)^{s}\left(\begin{array}{c}
n-1 \\
j
\end{array}\right)\left(\begin{array}{c}
n-j-1 \\
s
\end{array}\right) \mathbb{P}\left\{T_{1: s+j+1}>t\right\}
\end{aligned}
$$

and

$$
\begin{aligned}
& \mathbb{P}\left\{T_{m: n}>t \mid T_{i} \leq t\right\} \\
& \quad=\frac{1}{\mathbb{P}\left\{T_{i} \leq t\right\}} \sum_{j=n-m+1}^{n-1} \sum_{s=0}^{n-j}(-1)^{s}\left(\begin{array}{c}
n-1 \\
j
\end{array}\right)\left(\begin{array}{c}
n-j \\
s
\end{array}\right) \mathbb{P}\left\{T_{1: s+j}>t\right\},
\end{aligned}
$$

where $T_{1: i}=\min \left(T_{1}, \ldots, T_{i}\right)$ for $i \geq 1$.

Proof. It is clear that

$$
\begin{aligned}
\mathbb{P}\left\{T_{m: n}>t, T_{i}>t\right\} & \\
& =\sum_{j=n-m}^{n-1} \mathbb{P}\left\{j \text { of } T_{1}, \ldots, T_{i-1}, T_{i+1}, \ldots, T_{n} \text { is greater than } t, T_{i}>t\right\} .
\end{aligned}
$$


Because $T_{1}, \ldots, T_{n}$ are exchangeable,

$\mathbb{P}\left\{j\right.$ of $T_{1}, \ldots, T_{i-1}, T_{i+1}, \ldots, T_{n}$ is greater than $\left.t, T_{i}>t\right\}$

$$
=\left(\begin{array}{c}
n-1 \\
j
\end{array}\right) \mathbb{P}\left\{T_{1}>t, \ldots, T_{j}>t, T_{j+1} \leq t, \ldots, T_{n-1} \leq t, T_{i}>t\right\} .
$$

For a sequence of exchangeable binary variables $\xi_{1}, \ldots, \xi_{n}$, it is known that

$$
\begin{aligned}
\mathbb{P}\left\{\xi_{1}\right. & \left.=1, \ldots, \xi_{k}=1, \xi_{k+1}=0, \ldots, \xi_{n}=0\right\} \\
& =\sum_{s=0}^{n-k}(-1)^{s}\left(\begin{array}{c}
n-k \\
s
\end{array}\right) \mathbb{P}\left\{\xi_{1}=1, \ldots, \xi_{s+k}=1\right\} .
\end{aligned}
$$

Therefore,

$\mathbb{P}\left\{j\right.$ of $T_{1}, \ldots, T_{i-1}, T_{i+1}, \ldots, T_{n}$ is greater than $\left.t, T_{i}>t\right\}$

$$
\begin{aligned}
& =\left(\begin{array}{c}
n-1 \\
j
\end{array}\right) \mathbb{P}\left\{T_{1}>t, \ldots, T_{j}>t, T_{j+1} \leq t, \ldots, T_{n-1} \leq t, T_{i}>t\right\} \\
& =\left(\begin{array}{c}
n-1 \\
j
\end{array}\right) \sum_{s=0}^{n-j-1}(-1)^{s}\left(\begin{array}{c}
n-j-1 \\
s
\end{array}\right) \mathbb{P}\left\{T_{1}>t, \ldots, T_{s+j+1}>t\right\} .
\end{aligned}
$$

Thus, the proof of (7) is completed using (10) in (9). The proof of (8) is similar and, hence, omitted.

The following theorem reveals some properties of the vectors of coefficients $s^{+i}$ and $s^{-i}$.

Theorem 2. The following properties are satisfied for the vectors $s=\left(s_{1}(n), \ldots, s_{n}(n)\right)$, $\boldsymbol{s}^{+i}=\left(s_{1}^{+i}(n), \ldots, s_{n}^{+i}(n)\right)$, and $\boldsymbol{s}^{-i}=\left(s_{1}^{-i}(n), \ldots, s_{n}^{-i}(n)\right)$.

(i) The arithmetic mean of the mth component of $\boldsymbol{s}^{+i}$ over all $i=1, \ldots, n$ is equal to the mth element of the signature vector $s=\left(s_{1}(n), \ldots, s_{n}(n)\right)$, i.e.

$$
\frac{1}{n} \sum_{i=1}^{n} s_{m}^{+i}(n)=s_{m}(n) \text { for all } m=1, \ldots, n .
$$

(ii) For a fixed $i$,

$$
\sum_{m=1}^{n} s_{m}^{+i}(n)=1
$$

(iii) For a fixed $i$, if there is at least one $m$ such that $s_{m}^{-i}(n) \neq 0$, then

$$
\sum_{m=1}^{n} s_{m}^{-i}(n)=1
$$

Proof. From (3), we have

$$
\begin{aligned}
\sum_{i=1}^{n} s_{m}^{+i}(n) & =\sum_{i=1}^{n}\left[\frac{r_{n-m+1}^{+i}(n)}{\left(\begin{array}{c}
n-1 \\
n-m
\end{array}\right)}-\frac{r_{n-m}^{+i}(n)}{\left(\begin{array}{c}
n-1 \\
n-m-1
\end{array}\right)}\right] \\
& =\frac{1}{\left(\begin{array}{c}
n-1 \\
n-m
\end{array}\right)} \sum_{i=1}^{n} r_{n-m+1}^{+i}(n)-\frac{1}{\left(\begin{array}{c}
n-1 \\
n-m-1
\end{array}\right)} \sum_{i=1}^{n} r_{n-m}^{+i}(n)
\end{aligned}
$$




$$
\begin{aligned}
& =\frac{1}{\left(\begin{array}{c}
n-1 \\
n-m
\end{array}\right)}(n-m+1) r_{n-m+1}(n)-\frac{1}{\left(\begin{array}{c}
n-1 \\
n-m-1
\end{array}\right)}(n-m) r_{n-m}(n) \\
& =n\left[\frac{r_{n-m+1}(n)}{\left(\begin{array}{c}
n \\
n-m+1
\end{array}\right)}-\frac{r_{n-m}(n)}{\left(\begin{array}{c}
n \\
n-m
\end{array}\right)}\right] \\
& =n s_{m}(n) .
\end{aligned}
$$

Thus, Theorem 2(i) is proved. There is only one path set of size $n$ which includes all components, i.e. $r_{n}^{+i}(n)=1$. Therefore, the proof of Theorem 2(ii) follows since, from (3), we have

$$
\sum_{m=1}^{n} s_{m}^{+i}(n)=\frac{r_{n}^{+i}(n)}{\left(\begin{array}{l}
n-1 \\
n-1
\end{array}\right)}
$$

If $s_{m}^{-i}(n) \neq 0$ for at least one $m$ then there is at least one path set which does not include the $i$ th component. Such a path set is always the subset of the path set $\{1, \ldots, i-1, i+1, \ldots, n\}$. Therefore, $r_{n-1}^{-i}(n)=1$. The proof of Theorem 2(iii) follows from

$$
\sum_{m=1}^{n} s_{m}^{-i}(n)=\frac{r_{n-1}^{-i}(n)}{\left(\begin{array}{c}
n-1 \\
n-1
\end{array}\right)}
$$

Proposition 1. Let $\boldsymbol{s}^{+i}=\left(s_{1}^{+i}(n), \ldots, s_{n}^{+i}(n)\right)$ be the vector associated with the structure $\phi$, and $z^{-i}=\left(z_{1}^{-i}(n), \ldots, z_{n}^{-i}(n)\right)$ be the vector associated with the dual structure $\phi^{\mathrm{D}}$. Then

$$
z_{m}^{-i}(n)=s_{n-m+1}^{+i}(n) \text { for } m=2, \ldots, n, z_{1}^{-i}(n)=0 .
$$

Proof. From (3), we have

$$
s_{n-m+1}^{+i}(n)=\frac{r_{m}^{+i}(n)}{\left(\begin{array}{c}
n-1 \\
m-1
\end{array}\right)}-\frac{r_{m-1}^{+i}(n)}{\left(\begin{array}{c}
n-1 \\
m-2
\end{array}\right)} .
$$

If $d_{m}^{-i}(n)$ denotes the number of path sets which do not include $i$ th component for the dual structure $\phi^{\mathrm{D}}$, then

$$
r_{m}^{+i}(n)=\left(\begin{array}{c}
n-1 \\
m-1
\end{array}\right)-d_{n-m}^{-i}(n)
$$

Thus,

$$
s_{n-m+1}^{+i}(n)=\frac{d_{n-m+1}^{-i}(n)}{\left(\begin{array}{l}
n-1 \\
m-2
\end{array}\right)}-\frac{d_{n-m}^{-i}(n)}{\left(\begin{array}{l}
n-1 \\
m-1
\end{array}\right)}=z_{m}^{-i}(n) \quad \text { for } m=2, \ldots, n .
$$

By definition $z_{1}^{-i}(n)=0$.

In the special case of independent and identical components, the conditional probabilities in (2) become marginal probabilities of order statistics. The result is given in the following corollary.

Corollary 1. For a coherent system consisting of $n$ independent and identical components,

$$
I_{i}(t)=\sum_{m=1}^{n}\left[s_{m}^{+i}(n) \mathbb{P}\left\{T_{m: n-1}>t\right\}-s_{m}^{-i}(n) \mathbb{P}\left\{T_{m-1: n-1}>t\right\}\right]
$$

with $\mathbb{P}\left\{T_{n: n-1}>t\right\}=1$ and $\mathbb{P}\left\{T_{0: n-1}>t\right\}=0$. 


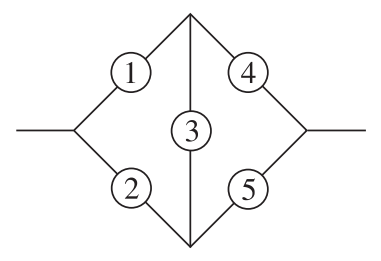

FIGURE 1: Bridge system.

Proof. If $T_{1}, \ldots, T_{n}$ are independent and identical then

$$
\mathbb{P}\left\{T_{m: n}>t \mid T_{i}>t\right\}=\mathbb{P}\left\{T_{m: n-1}>t\right\}, \quad \mathbb{P}\left\{T_{m: n}>t \mid T_{i} \leq t\right\}=\mathbb{P}\left\{T_{m-1: n-1}>t\right\} .
$$

Thus, the proof follows from Theorem 1.

Example 1. Consider the bridge system depicted in Figure 1. For this system

$$
\begin{gathered}
s^{+i}=\left(0, \frac{1}{6}, \frac{7}{12}, \frac{1}{4}, 0\right), \quad s^{-i}=\left(0, \frac{1}{4}, \frac{7}{12}, \frac{1}{6}, 0\right) \quad \text { for } i=1,2,4,5, \\
s^{+3}=\left(0, \frac{1}{3}, \frac{2}{3}, 0,0\right), \quad s^{-3}=(0,0,1,0,0) .
\end{gathered}
$$

Using Theorem 2, the signature of this bridge system is $s=\left(0, \frac{1}{5}, \frac{3}{5}, \frac{1}{5}, 0\right)$. From Theorem 1 ,

$$
\begin{aligned}
I_{i}(t)= & {\left[\frac{1}{6} \mathbb{P}\left\{T_{2: 5}>t \mid T_{i}>t\right\}+\frac{7}{12} \mathbb{P}\left\{T_{3: 5}>t \mid T_{i}>t\right\}+\frac{1}{4} \mathbb{P}\left\{T_{4: 5}>t \mid T_{i}>t\right\}\right] } \\
& -\left[\frac{1}{4} \mathbb{P}\left\{T_{2: 5}>t \mid T_{i} \leq t\right\}+\frac{7}{12} \mathbb{P}\left\{T_{3: 5}>t \mid T_{i} \leq t\right\}+\frac{1}{6} \mathbb{P}\left\{T_{4: 5}>t \mid T_{i} \leq t\right\}\right]
\end{aligned}
$$

for $i=1,2,4,5$, and

$$
I_{3}(t)=\frac{1}{3} \mathbb{P}\left\{T_{2: 5}>t \mid T_{3}>t\right\}+\frac{2}{3} \mathbb{P}\left\{T_{3: 5}>t \mid T_{3}>t\right\}-\mathbb{P}\left\{T_{3: 5}>t \mid T_{3} \leq t\right\} .
$$

The computation of the elements of the vectors $s, s^{+i}$, and $s^{-i}$ requires the numbers $r_{m}(n)$, $r_{m}^{+i}(n)$, and $r_{m}^{-i}(n)$ for a given structure and $i$. Although these numbers can be obtained by finding all path sets of the structure for coherent systems with few components, their computation for a system with arbitrary number of components is a well-defined combinatorial problem. In Table 1 we present values for the vectors $\boldsymbol{s}^{+i}$ and $\boldsymbol{s}^{-i}, i=1, \ldots, n$ for all coherent systems of order $n=3$. (See Eryilmaz (2015) for the table of results for the $n=4$ case.) The elements of these vectors are computed by listing all path sets of coherent systems with $n=3$ and $n=4$ components. On the other hand, in the following example we illustrate the computation of the numbers $r_{m}(n), r_{m}^{+i}(n)$, and $r_{m}^{-i}(n)$ for a specific structure using combinatorial arguments.

Example 2. Assume that the system has a linear consecutive- $k$-out-of- $n$ : $F$ structure, i.e. it consists of $n$ linearly ordered components, and fails if and only if at least $k$ consecutive components fail. For this structure, the number of path sets of size $m$ is given by

$$
r_{m}(n)=\sum_{j=0}^{\min (m+1,[(n-m) / k])}(-1)^{j}\left(\begin{array}{c}
m+1 \\
j
\end{array}\right)\left(\begin{array}{c}
n-j k \\
m
\end{array}\right) ;
$$

see, e.g. Eryilmaz (2010). Under the condition that the $i$ th component is in a working state, the linear consecutive- $k$-out-of- $n: F$ system can be decomposed into two modules, where the first 
TABLE 1: The vectors $\boldsymbol{s}, \boldsymbol{s}^{+i}$, and $\boldsymbol{s}^{-i}$ for all coherent systems of order $n=3$.

\begin{tabular}{|c|c|c|c|}
\hline System type & $i$ & $s^{+i}$ & $\mathbf{s}^{-i}$ \\
\hline \multicolumn{4}{|c|}{ Series. $s=(1,0,0)$} \\
\hline & 1 & $(1,0,0)$ & $(0,0,0)$ \\
\hline & 2 & $(1,0,0)$ & $(0,0,0)$ \\
\hline & 3 & $(1,0,0)$ & $(0,0,0)$ \\
\hline \multicolumn{4}{|c|}{$\min \left(T_{2}, \max \left(T_{1}, T_{3}\right)\right) . s=\left(\frac{1}{3}, \frac{2}{3}, 0\right)$} \\
\hline & 1 & $\left(\frac{1}{2}, \frac{1}{2}, 0\right)$ & $(0,1,0)$ \\
\hline & 2 & $(0,1,0)$ & $(0,0,0)$ \\
\hline & 3 & $\left(\frac{1}{2}, \frac{1}{2}, 0\right)$ & $(0,1,0)$ \\
\hline \multicolumn{4}{|c|}{ 2-out-of-3:F.s $=(0,1,0)$} \\
\hline & 1 & $(0,1,0)$ & $(0,1,0)$ \\
\hline & 2 & $(0,1,0)$ & $(0,1,0)$ \\
\hline & 3 & $(0,1,0)$ & $(0,1,0)$ \\
\hline \multicolumn{4}{|c|}{$\max \left(T_{2}, \min \left(T_{1}, T_{3}\right)\right) . s=\left(0, \frac{2}{3}, \frac{1}{3}\right)$} \\
\hline & 1 & $(0,1,0)$ & $\left(0, \frac{1}{2}, \frac{1}{2}\right)$ \\
\hline & 2 & $(0,0,1)$ & $(0,1,0)$ \\
\hline & 3 & $(0,1,0)$ & $\left(0, \frac{1}{2}, \frac{1}{2}\right)$ \\
\hline \multicolumn{4}{|c|}{ Parallel. $s=(0,0,1)$} \\
\hline & 1 & $(0,0,1)$ & $(0,0,1)$ \\
\hline & 2 & $(0,0,1)$ & $(0,0,1)$ \\
\hline & 3 & $(0,0,1)$ & $(0,0,1)$ \\
\hline
\end{tabular}

(second) module consists of $i-1(n-i)$ components. Assume that the first module includes $m_{1}$ working components. Then the second module includes $m-m_{1}-1$ components. Thus,

$$
r_{m}^{+i}(n)=\sum_{m_{1}=\max (0, m-n+i-1)}^{\min (i-1, m-1)} r_{m_{1}}(i-1) r_{m-m_{1}-1}(n-i), \quad r_{m}^{-i}(n)=r_{m}(n)-r_{m}^{+i}(n)
$$

\section{Series system with two modules}

Consider a coherent system consisting of $n=n_{1}+n_{2}$ components with the index set of components $C=\left\{1, \ldots, n_{1}+n_{2}\right\}$. Suppose that the system with the component index set $C$ consists of two modules with respective component index sets $\left\{1, \ldots, n_{1}\right\}$ and $\left\{n_{1}+1, \ldots, n_{1}+\right.$ $\left.n_{2}\right\}$ and structure functions $\phi_{1}$ and $\phi_{2}$. If the overall system has a series structure, i.e. the disjoint modules are serially connected, then the system's lifetime is represented as

$$
T_{*}=\min \left(\phi_{1}\left(T_{1}, \ldots, T_{n_{1}}\right), \phi_{2}\left(T_{n_{1}+1}, \ldots, T_{n}\right)\right) .
$$

Let $k_{i}$ denote the minimum number of working components for the functioning of the $i$ th module, $i=1,2$. If $h_{m}(n)$ denotes the number of path sets of size $m$ of the system with the 
lifetime $T_{*}$, then

$$
h_{m}(n)=\sum_{j=\max \left(k_{1}, m-n_{2}\right)}^{\min \left(m-k_{2}, n_{1}\right)} u_{j}\left(n_{1}\right) v_{m-j}\left(n_{2}\right) \quad \text { for } m \geq k_{1}+k_{2},
$$

where $u_{m}\left(n_{1}\right)$ and $v_{m}\left(n_{2}\right)$ represent respectively the number of path sets of size $m$ of the structures $\phi_{1}$ and $\phi_{2}$; see Eryilmaz (2014).

In this section we aim to derive formulae for the vectors $s^{+i}$ and $s^{-i}$ associated with the system (11) based on the corresponding vectors of the structures $\phi_{1}$ and $\phi_{2}$.

In the following theorem, we obtain an expression for the number of path sets of size $m$ including the $i$ th component for the system with lifetime $T_{*}$ in terms of the number of path sets of the structures $\phi_{1}$ and $\phi_{2}$. Denote by $u_{m}^{+i}\left(n_{1}\right)\left(v_{m}^{+i}\left(n_{2}\right)\right)$ the number of path sets of size $m$ including the $i$ th component for the system with structure $\phi_{1}\left(\phi_{2}\right)$.

Theorem 3. The number of path sets of size $m$ including the ith component for the system with lifetime $T_{*}$ is

$$
r_{m}^{+i}(n)= \begin{cases}\sum_{\substack{\max \left(k_{1}^{+i}, m-n_{2}\right) \\ \min \left(n_{2}, m-k_{1}\right)}}^{\min \left(n_{1}, m-k_{2}\right)} u_{j}^{+i}\left(n_{1}\right) v_{m-j}\left(n_{2}\right) \quad \text { if } i \in\left\{1, \ldots, n_{1}\right\}, \\ \sum_{l=\max \left(k_{2}^{+i}, m-n_{1}\right)} u_{m-l}\left(n_{1}\right) v_{l}^{+i}\left(n_{2}\right) \quad \text { if } i \in\left\{n_{1}+1, \ldots, n_{1}+n_{2}\right\},\end{cases}
$$

where $k_{1}^{+i}\left(k_{2}^{+i}\right)$ is the size of the path set which has the smallest size and includes the ith component for the system with structure $\phi_{1}\left(\phi_{2}\right)$.

Proof. For $i \in\left\{1, \ldots, n_{1}\right\}$,

$$
r_{m}^{+i}(n)=\sum_{\substack{j+l=m \\ j \geq k_{1}^{+i}, l \geq k_{2}}} u_{j}^{+i}\left(n_{1}\right) v_{l}\left(n_{2}\right),
$$

which follows from the fact that both modules must work for the series system to function and that the first module works and includes $j$ working components being the $i$ th component in a functioning state, and the second module works and includes $l$ working components such that $j \geq k_{1}^{+i}, l \geq k_{2}$, and $j+l=m$. Thus, we have

$$
r_{m}^{+i}(n)=\sum_{j=\max \left(k_{1}^{+i}, m-n_{2}\right)}^{\min \left(n_{1}, m-k_{2}\right)} u_{j}^{+i}\left(n_{1}\right) v_{m-j}\left(n_{2}\right) \quad \text { for } i \in\left\{1, \ldots, n_{1}\right\} .
$$

The case when the $i$ th component belongs to the second module can be proved similarly.

The following result is immediate since for any coherent system $r_{m}^{+i}(n)+r_{m}^{-i}(n)=r_{m}(n)$.

Corollary 2. The number of path sets of size $m$ which do not include the ith component for the system with lifetime $T_{*}$ is

$$
r_{m}^{-i}(n)=\sum_{j=\max \left(k_{1}, m-n_{2}\right)}^{\min \left(m-k_{2}, n_{1}\right)} u_{j}\left(n_{1}\right) v_{m-j}\left(n_{2}\right)-r_{m}^{+i}(n),
$$

where $r_{m}^{+i}(n)$ is given by Theorem 3 for $i=, \ldots, n$. 
Let $\boldsymbol{p}=\left(p_{1}\left(n_{1}\right), \ldots, p_{n_{1}}\left(n_{1}\right)\right)$ and $\boldsymbol{q}=\left(q_{1}\left(n_{2}\right), \ldots, q_{2}\left(n_{2}\right)\right)$ denote the signatures of the structures $\phi_{1}$ and $\phi_{2}$, respectively. Then

$$
u_{j}\left(n_{1}\right)=\left(\begin{array}{c}
n_{1} \\
j
\end{array}\right) \sum_{a=n_{1}-j+1}^{n_{1}} p_{a}\left(n_{1}\right), \quad v_{l}\left(n_{2}\right)=\left(\begin{array}{c}
n_{2} \\
l
\end{array}\right) \sum_{b=n_{2}-l+1}^{n_{2}} q_{b}\left(n_{2}\right) .
$$

On the other hand,

$$
u_{j}^{+i}\left(n_{1}\right)=\left(\begin{array}{c}
n_{1}-1 \\
j-1
\end{array}\right) \sum_{a=n_{1}-j+1}^{n_{1}} p_{a}^{+i}\left(n_{1}\right) \quad \text { for } i \in\left\{1, \ldots, n_{1}\right\}
$$

and

$$
v_{l}^{+i}\left(n_{2}\right)=\left(\begin{array}{c}
n_{2}-1 \\
l-1
\end{array}\right) \sum_{b=n_{2}-l+1}^{n_{2}} q_{b}^{+i}\left(n_{2}\right) \quad \text { for } i \in\left\{n_{1}+1, \ldots, n_{1}+n_{2}\right\} .
$$

Using (13)-(15) in Theorem 3, we have

$$
\begin{aligned}
r_{m}^{+i}(n)= & \sum_{j=\max \left(k_{1}^{+i}, m-n_{2}\right)}^{\min \left(n_{1}, m-k_{2}\right)}\left(\begin{array}{c}
n_{1}-1 \\
j-1
\end{array}\right) \sum_{a=n_{1}-j+1}^{n_{1}} p_{a}^{+i}\left(n_{1}\right)\left(\begin{array}{c}
n_{2} \\
m-j
\end{array}\right) \\
& \times \sum_{b=n_{2}-m+j+1}^{n_{2}} q_{b}\left(n_{2}\right) \quad \text { for } i \in\left\{1, \ldots, n_{1}\right\}
\end{aligned}
$$

and

$$
\begin{aligned}
r_{m}^{+i}(n)= & \sum_{l=\max \left(k_{2}^{+i}, m-n_{1}\right)}^{\min \left(n_{2}, m-k_{1}\right)}\left(\begin{array}{c}
n_{1} \\
m-l
\end{array}\right) \sum_{a=n_{1}-m+l+1}^{n_{1}} p_{a}\left(n_{1}\right)\left(\begin{array}{c}
n_{2}-1 \\
l-1
\end{array}\right) \\
& \times \sum_{b=n_{2}-l+1}^{n_{2}} q_{b}^{+i}\left(n_{2}\right) \quad \text { for } i \in\left\{n_{1}+1, \ldots, n_{1}+n_{2}\right\} .
\end{aligned}
$$

Therefore, using (3), the vector of coefficients $s^{+i}=\left(s_{1}^{+i}(n), \ldots, s_{n}^{+i}(n)\right)$ for the system with lifetime $T_{*}$ is found to be

$$
\begin{aligned}
s_{m}^{+i}(n)= & \frac{1}{\left(\begin{array}{c}
n-1 \\
n-m
\end{array}\right)} \sum_{j=\max \left(k_{1}^{+i}, n-n_{2}-m+1\right)}^{\min \left(n_{1}, n-k_{2}-m+1\right)}\left(\begin{array}{c}
n_{1}-1 \\
j-1
\end{array}\right) \sum_{a=n_{1}-j+1}^{n_{1}} p_{a}^{+i}\left(n_{1}\right) \\
& \times\left(\begin{array}{c}
n_{2} \\
n-m-j+1
\end{array}\right) \sum_{b=n_{2}+m+j-n}^{n_{2}} q_{b}\left(n_{2}\right) \\
& -\frac{1}{\left(\begin{array}{c}
n-1 \\
n-m-1
\end{array}\right)} \sum_{j=\max \left(k_{1}^{+i}, n-n_{2}-m\right)}^{\min \left(n_{1}, n-k_{2}-m\right)}\left(\begin{array}{c}
n_{1}-1 \\
j-1
\end{array}\right) \sum_{a=n_{1}-j+1}^{n_{1}} p_{a}^{+i}\left(n_{1}\right) \\
& \times\left(\begin{array}{c}
n_{2} \\
n-m-j
\end{array}\right) \sum_{b=n_{2}+m+j-n+1}^{n_{2}} q_{b}\left(n_{2}\right) \quad \text { for } m=1, \ldots, n, i \in\left\{1, \ldots, n_{1}\right\} .
\end{aligned}
$$


Similarly, for $i \in\left\{n_{1}+1, \ldots, n_{1}+n_{2}\right\}$,

$$
\begin{aligned}
s_{m}^{+i}(n)= & \frac{1}{\left(\begin{array}{c}
n-1 \\
n-m
\end{array}\right)} \sum_{l=\max \left(k_{2}^{+i}, n-m-n_{1}+1\right)}^{\min \left(n_{2}, n-m-k_{1}+1\right)}\left(\begin{array}{c}
n_{1} \\
n-m-l+1
\end{array}\right) \sum_{a=n_{1}-n+m+l}^{n_{1}} p_{a}\left(n_{1}\right) \\
& \times\left(\begin{array}{c}
n_{2}-1 \\
l-1
\end{array}\right) \sum_{b=n_{2}-l+1}^{n_{2}} q_{b}^{+i}\left(n_{2}\right) \\
& -\frac{1}{\left(\begin{array}{c}
n-1 \\
n-m-1
\end{array}\right)} \sum_{l=\max \left(k_{2}^{+i}, n-m-n_{1}\right)}^{\min \left(n_{2}, n-m-k_{1}\right)}\left(\begin{array}{c}
n_{1} \\
n-m-l
\end{array}\right) \sum_{a=n_{1}-n+m+l+1}^{n_{1}} p_{a}\left(n_{1}\right) \\
& \times\left(\begin{array}{c}
n_{2}-1 \\
l-1
\end{array}\right) \sum_{b=n_{2}-l+1}^{n_{2}} q_{b}^{+i}\left(n_{2}\right) \quad \text { for } m=1, \ldots, n .
\end{aligned}
$$

Thus, the vector of coefficients $\boldsymbol{s}^{+i}=\left(s_{1}^{+i}(n), \ldots, s_{n}^{+i}(n)\right)$ for the system with lifetime $T_{*}$ can be computed using the signature vectors

$$
\begin{array}{cc}
\boldsymbol{p}=\left(p_{1}\left(n_{1}\right), \ldots, p_{n_{1}}\left(n_{1}\right)\right), & \boldsymbol{q}=\left(q_{1}\left(n_{2}\right), \ldots, q_{2}\left(n_{2}\right)\right), \\
\boldsymbol{p}^{+i}=\left(p_{1}^{+i}\left(n_{1}\right), \ldots, p_{n_{1}}^{+i}\left(n_{1}\right)\right), & \boldsymbol{q}^{+i}=\left(q_{1}^{+i}\left(n_{2}\right), \ldots, q_{n_{2}}^{+i}\left(n_{2}\right)\right) .
\end{array}
$$

Using Corollary 2 with (13), (16), and (17), we obtain

$$
\begin{aligned}
r_{m}^{-i}(n)= & \sum_{j=\max \left(k_{1}, m-n_{2}\right)}^{\min \left(m-k_{2}, n_{1}\right)}\left(\begin{array}{c}
n_{1} \\
j
\end{array}\right) \sum_{a=n_{1}-j+1}^{n_{1}} p_{a}\left(n_{1}\right)\left(\begin{array}{c}
n_{2} \\
m-j
\end{array}\right) \sum_{b=n_{2}-m+j+1}^{n_{2}} q_{b}\left(n_{2}\right) \\
& -\sum_{j=\max \left(k_{1}^{+i}, m-n_{2}\right)}^{\min \left(n_{1}, m-k_{2}\right)}\left(\begin{array}{c}
n_{1}-1 \\
j-1
\end{array}\right) \sum_{a=n_{1}-j+1}^{n_{1}} p_{a}^{+i}\left(n_{1}\right)\left(\begin{array}{c}
n_{2} \\
m-j
\end{array}\right) \sum_{b=n_{2}-m+j+1}^{n_{2}} q_{b}\left(n_{2}\right)
\end{aligned}
$$

for $i \in\left\{1, \ldots, n_{1}\right\}$, and

$$
\begin{aligned}
r_{m}^{-i}(n)= & \sum_{j=\max \left(k_{1}, m-n_{2}\right)}^{\min \left(m-k_{2}, n_{1}\right)}\left(\begin{array}{c}
n_{1} \\
j
\end{array}\right) \sum_{a=n_{1}-j+1}^{n_{1}} p_{a}\left(n_{1}\right)\left(\begin{array}{c}
n_{2} \\
m-j
\end{array}\right) \sum_{b=n_{2}-m+j+1}^{n_{2}} q_{b}\left(n_{2}\right) \\
& -\sum_{l=\max \left(k_{2}^{+i}, m-n_{1}\right)}^{\min \left(n_{2}, m-k_{1}\right)}\left(\begin{array}{c}
n_{1} \\
m-l
\end{array}\right) \sum_{a=n_{1}-m+l+1}^{n_{1}} p_{a}\left(n_{1}\right)\left(\begin{array}{c}
n_{2}-1 \\
l-1
\end{array}\right) \sum_{b=n_{2}-l+1}^{n_{2}} q_{b}^{+i}\left(n_{2}\right)
\end{aligned}
$$

for $i \in\left\{n_{1}+1, \ldots, n_{1}+n_{2}\right\}$.

Thus, using (4), the vector of coefficients $\boldsymbol{s}^{-i}=\left(s_{1}^{-i}(n), \ldots, s_{n}^{-i}(n)\right)$ for the system with lifetime $T_{*}$ can be computed using the signature vectors

$$
\begin{array}{cc}
\boldsymbol{p}=\left(p_{1}\left(n_{1}\right), \ldots, p_{n_{1}}\left(n_{1}\right)\right), & \boldsymbol{q}=\left(q_{1}\left(n_{2}\right), \ldots, q_{2}\left(n_{2}\right)\right), \\
\boldsymbol{p}^{+i}=\left(p_{1}^{+i}\left(n_{1}\right), \ldots, p_{n_{1}}^{+i}\left(n_{1}\right)\right), & \boldsymbol{q}^{+i}=\left(q_{1}^{+i}\left(n_{2}\right), \ldots, q_{n_{2}}^{+i}\left(n_{2}\right)\right) .
\end{array}
$$




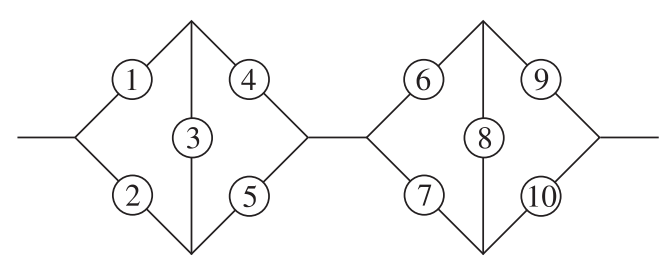

FIGURE 2: Series composition of two bridge structures

Example 3. Consider the series composition of the bridge structures as shown in Figure 2. From Example 1, the signatures of the modules are $\boldsymbol{p}=\left(0, \frac{1}{5}, \frac{3}{5}, \frac{1}{5}, 0\right)$ and $\boldsymbol{q}=\left(0, \frac{1}{5}, \frac{3}{5}, \frac{1}{5}, 0\right)$, and

$$
\begin{gathered}
\boldsymbol{p}^{+i}=\left(0, \frac{1}{6}, \frac{7}{12}, \frac{1}{4}, 0\right), \quad \boldsymbol{p}^{-i}=\left(0, \frac{1}{4}, \frac{7}{12}, \frac{1}{6}, 0\right) \quad \text { for } i=1,2,4,5, \\
\boldsymbol{p}^{+3}=\left(0, \frac{1}{3}, \frac{2}{3}, 0,0\right), \quad \boldsymbol{p}^{-3}=(0,0,1,0,0),
\end{gathered}
$$

and

$$
\begin{gathered}
\boldsymbol{q}^{+i}=\left(0, \frac{1}{6}, \frac{7}{12}, \frac{1}{4}, 0\right), \quad \boldsymbol{q}^{-i}=\left(0, \frac{1}{4}, \frac{7}{12}, \frac{1}{6}, 0\right) \quad \text { for } i=6,7,9,10, \\
\boldsymbol{q}^{+8}=\left(0, \frac{1}{3}, \frac{2}{3}, 0,0\right), \quad \boldsymbol{q}^{-8}=(0,0,1,0,0) .
\end{gathered}
$$

By (19) and (20), we obtain

$$
s^{+i}=\left(0, \frac{1}{12}, \frac{17}{84}, \frac{37}{126}, \frac{5}{18}, \frac{5}{42}, \frac{1}{42}, 0,0,0\right) \quad \text { for } i=1,2,4,5,6,7,9,10,
$$

and

$$
\boldsymbol{s}^{+i}=\left(0, \frac{1}{9}, \frac{31}{126}, \frac{41}{126}, \frac{16}{63}, \frac{4}{63}, 0,0,0,0\right) \text { for } i=3,8 .
$$

From Theorem 2, the signature of the overall series system is obtained as

$$
s=\left(0, \frac{4}{45}, \frac{19}{90}, \frac{3}{10}, \frac{86}{315}, \frac{34}{315}, \frac{2}{105}, 0,0,0,\right)
$$

which coincides with the result in Da et al. (2012). Substituting (20) and (21) into (4), we obtain

$$
s^{-i}=\left(0, \frac{1}{9}, \frac{2}{9}, \frac{25}{84}, \frac{65}{252}, \frac{2}{21}, \frac{1}{63}, 0,0,0\right) \text { for } i=1,2,4,5,6,7,9,10
$$

and

$$
s^{-i}=\left(0,0, \frac{2}{9}, \frac{47}{126}, \frac{43}{126}, \frac{2}{63}, \frac{2}{63}, 0,0,0\right) \quad \text { for } i=3,8
$$

\section{Acknowledgement}

The author is grateful to the anonymous referee for his/her useful comments which improved the presentation of this paper.

\section{References}

ANDREws, J. D. (2008). Birnbaum and criticality measures of component contribution to the failure of phased missions. Reliab. Eng. System Safety 93, 1861-1866.

Barlow, R. E. And Proschan, F. (1975). Importance of system components and fault tree events. Stoch. Process. Appl. 3, 153-173.

Bergman, B. (1985). On some new reliability importance measures. In Safety of Computer Control Systems (Proc. SAFECOMP' 85; Como, Italy), pp. 61-64. 
Birnbaum, Z. W. (1969). On the importance of different components in a multicomponent system. In Multivariate Analysis, II, Academic Press, New York, pp. 581-592.

Boland, P. J. (2001). Signatures of indirect majority systems. J. Appl. Prob. 38, 597-603.

Boland, P. J AND El-Neweihi, E. (1995). Measures of component importance in reliability theory. Comput. Operat. Res. 22, 455-463.

Butler, D. A. (1977). An importance ranking for system components based upon cuts. Operat. Res. 25, 874-879.

DA, G., Zheng, B. ANd Hu, T. (2012). On computing signatures of coherent systems. J. Multivariate Anal. 103, $142-150$.

Eryilmaz, S. (2015). Table of results for vectors $s^{+i}, s^{-i}$ for all coherent systems of order $n=4$. Available at https://www.dropbox.com/s/krc2gw7pmeegnzp/table2.pdf.

Eryilmaz, S. (2010). Review of recent advances in reliability of consecutive $k$-out-of- $n$ and related systems. J. Risk Reliab. 224, 225-237.

ERyilmaz, S. (2013). On residual lifetime of coherent systems after the $r$ th failure. Statist. Papers 54, $243-250$.

ERYILMAZ, S. (2014). On signatures of series and parallel systems consisting of modules with arbitrary structures. Commun. Statist. Simul. Comput. 43, 1202-1211.

Eryilmaz, S., Koutras, M. V. And Triantafyllou, I. S. (2011). Signature based analysis of $m$-consecutive- $k$-outof- $n$ : $F$ systems with exchangeable components. Naval Res. Logistics 58, 344-354.

Hwang, F. K. (2001). A new index of component importance. Operat. Res. Lett. 28, 75-79.

IYER, S. (1992). The Barlow-Proschan importance and its generalizations with dependent components. Stoch. Process. Appl. 42, 353-359.

Kuo, W. AND ZHU, X. (2012). Some recent advances on importance measures in reliability. IEEE Trans. Reliab. 61, 344-360.

Kuo, W. And Zuo, M. J. (2003). Optimal Reliability Modeling: Principles and Applications. John Wiley, Chichester.

Natvig, B. (1979). A suggestion of a new measure of importance of system components. Stoch. Process. Appl. 9, 319-330.

Natvig, B. (1985). New light on measures of importance of system components. Scand. J. Statist. 12, $43-54$.

NATVig, B. (2011). Measures of component importance in nonrepairable and repairable multistate strongly coherent systems. Method. Comput. Appl. Prob. 13, 523-547.

Natvig, B. And GÅsemyr, J. (2009). New results on the Barlow-Proschan and Natvig measures of component importance in nonrepairable and repairable systems. Method. Comput. Appl. Prob. 11, 603-620.

Navarro, J. AND Rubio, R. (2011). A note on necessary and sufficient conditions for ordering properties of coherent systems with exchangeable components. Naval Res. Logistics 58, 478-489.

NAVARRo, J., RyCHLIK, T. (2007). Reliability and expectation bounds for coherent systems with exchangeable components. J. Multivariate Anal. 98, 102-113.

Navarro, J. ANd Spizzichino, F. (2010). Comparisons of series and parallel systems with components sharing the same copula. Appl. Stoch. Models Business Industry 26, 775-791.

Navarro, J., Ruiz, J.M. and Sandoval, C. J. (2005). A note on comparisons among coherent systems with dependent components using signatures. Statist. Prob. Lett. 72, 179-185.

Navarro, J., Samaniego, F. J. and Balakrishnan, N. (2013). Mixture representations for the joint distribution of lifetimes of two coherent systems with shared components. Adv. Appl. Prob. 45, 1011-1027.

Navarro, J., Samaniego, F. J., Balakrishnan, N. and Bhattacharya, D. (2008). On the application and extension of system signatures in engineering reliability. Naval Res. Logistics 55, 313-327.

Parvardeh, A., Balakrishnan, N. (2013). Conditional residual lifetimes of coherent systems. Statist. Prob. Lett. 83, 2664-2672.

Samaniego, F. J. (2007). System Signatures and Their Applications in Engineering Reliability. Springer, New York.

Triantafyllou, I. S. AND Koutras, M. V. (2014). Reliability properties of ( $n . f, k)$ systems, IEEE Trans. Reliability 63, 357-366.

XIE, M. (1987). On some importance measures of system components. Stoch. Process. Appl. 25, 273-280.

Xie, M. And Bergman, B. (1991). On a general measure of component importance. J. Statist. Planning Inference 29, 211-220.

Zarezadeh, S., Asadi, M. And Balakrishnan, N. (2014). Dynamic network reliability modeling under nonhomogeneous Poisson processes. Europ. J. Operat. Res. 232, 561-571.

Zhu, X. And Kuo, W. (2014). Importance measures in reliability and mathematical programming. Ann. Operat. Res. 212, 241-267. 